University of Wollongong

Research Online

Faculty of Engineering and Information

Faculty of Engineering and Information

Sciences - Papers: Part A

Sciences

$1-1-2014$

Dynamic adjustment of OLTC parameters using voltage sensitivity while utilizing DG for Volt/VAr support

Dothinka Ranamuka Rallage

University of Wollongong, dssrr987@uowmail.edu.au

Ashish Agalgaonkar

University of Wollongong, ashish@uow.edu.au

Kashem M. Muttaqi

University of Wollongong, kashem@uow.edu.au

Follow this and additional works at: https://ro.uow.edu.au/eispapers

Part of the Engineering Commons, and the Science and Technology Studies Commons

Research Online is the open access institutional repository for the University of Wollongong. For further information contact the UOW Library: research-pubs@uow.edu.au 


\title{
Dynamic adjustment of OLTC parameters using voltage sensitivity while utilizing DG for Volt/VAr support
}

\author{
Abstract \\ Voltage regulation in medium voltage (MV) distribution systems is still widely performed with the aid of \\ substation on-load tap changer (OLTC). An OLTC can be operated with or without enacting the line drop \\ compensation (LDC) module embedded in the OLTC control. However, in case of distribution systems \\ with higher penetration of renewable and distributed generation (DG), it is well known that the DG reverse \\ power flow can increase the number of OLTC tap operations under certain system conditions. On the \\ other hand, voltage correction by DG is one of the promising concepts highly regarded by the researchers \\ and engineers. In this paper, a methodology is proposed for Volt/VAr support by means of DG and \\ dynamic adjustment of OLTC parameters. As an assessment tool, first order sensitivity of the regulating \\ point voltage, estimated by LDC scheme, to change in reactive power support provided by voltage support \\ DG is derived. The simulation studies are carried out on a test system using MATLAB software, and \\ results have demonstrated the accuracy of proposed sensitivity and its application for dynamically \\ updating the OLTC parameters for ensuring effective voltage control with the aid of synchronous machine \\ based voltage support DG.

\section{Disciplines} \\ Engineering | Science and Technology Studies

\section{Publication Details} \\ D. Ranamuka, A. P. Agalgaonkar \& K. M. Muttaqi , "Dynamic adjustment of OLTC parameters using voltage \\ sensitivity while utilizing DG for Volt/VAr support," in IEEE Power \& Energy Society General Meeting, 2014, \\ pp. 1-5.
}




\title{
Dynamic Adjustment of OLTC Parameters using Voltage Sensitivity while utilizing DG for Volt/VAr Support
}

\author{
D. Ranamuka, Student Member, IEEE, A. P. Agalgaonkar, Senior Member, IEEE, and K. M. Muttaqi, \\ Senior Member, IEEE
}

\begin{abstract}
Voltage regulation in medium voltage (MV) distribution systems is still widely performed with the aid of substation on-load tap changer (OLTC). An OLTC can be operated with or without enacting the line drop compensation (LDC) module embedded in the OLTC control. However, in case of distribution systems with higher penetration of renewable and distributed generation (DG), it is well known that the DG reverse power flow can increase the number of OLTC tap operations under certain system conditions. On the other hand, voltage correction by DG is one of the promising concepts highly regarded by the researchers and engineers. In this paper, a methodology is proposed for Volt/VAr support by means of DG and dynamic adjustment of OLTC parameters. As an assessment tool, first order sensitivity of the regulating point voltage, estimated by LDC scheme, to change in reactive power support provided by voltage support DG is derived. The simulation studies are carried out on a test system using MATLAB software, and results have demonstrated the accuracy of proposed sensitivity and its application for dynamically updating the OLTC parameters for ensuring effective voltage control with the aid of synchronous machine based voltage support DG.
\end{abstract}

Index Terms--distribution system; line drop compensation; onload tap changer; voltage regulation; voltage support distributed generation.

\section{INTRODUCTION}

A CCORDING to the IEEE Application Guide for IEEE Standard 1547, the general guidelines state that distributed generation (DG) units are expected to be operated at or near unity power factor to avoid actively controlling the system voltage. However, in cases where in the DG units may significantly affect the network parameters, they can be operated at a specific power factor. Numerous strategies and controls have been proposed in the literature for operating both renewable and non-renewable DG units for system voltage support. The DG reactive power can be controlled by exporting and absorbing reactive power (VAr) for maximizing the voltage support.

The authors are with the Australian Power Quality and Reliability Centre, School of Electrical, Computer and Telecommunications Engineering, University of Wollongong, New South Wales 2522 Australia. (e-mail: dssrr987@uowmail.edu.au; ashish@uow.edu.au; kashem@uow.edu.au)

*This paper was selected for presentation session of the Best Conference Papers on Integrated Power System Operations in IEEE-PES General Meeting, 2014, Washington DC.
In [1], a voltage control strategy which is designed for maximizing the DG voltage support is presented. In the controller design discussed in [1], there is an adaptive control strategy to utilize DG power factor control mode operation for voltage support. A reactive power control scheme is proposed in order to utilize a doubly fed induction generator (DFIG) for voltage support in [2]. Also in [3], a strategy is proposed for voltage control by direct active and reactive power control of grid connected DFIG based wind system using sliding mode control principle. A local control scheme is proposed for voltage regulation by a DFIG based wind system in [4]. The controller proposed in [4] provides adjustments to reactive power based on the active power generated by the DFIG unit, in order to support the system voltage. In [5], various design options for controlling the reactive power generated by photovoltaic (PV) units have been tested. It is aimed to utilize PV systems for voltage support against the voltage variations caused by varying irradiance conditions. A control strategy is proposed for night time application of PV solar farms to support the grid voltage in [6]. It utilizes the PV solar farm as a static synchronous compensator for voltage support, when PV farm does not produce any active power. In [7], a control strategy is designed for maximizing and prioritizing the DG voltage support. Also, a practical implementation strategy for proposed control is presented in [7], which is aided by a distribution management system (DMS).

In this paper, a methodology is proposed to assess the DG impact on voltage correction by on-load tap changer (OLTC) through sensitivity analysis, and accordingly update the line drop compensation (LDC) settings for effective voltage control assuming DG units are operated for voltage support in the network. The reactive power capability of different DG units (i.e., synchronous machine based DG, DFIG and PV units) for voltage support has been detailed in [8]. MATLAB is used for modeling and simulation, and the results are presented.

\section{DG IMPACT ON VOLTAGE CORRECTION BY OLTC}

An OLTC enabled with LDC can be used to control the voltage at a remote regulating point (load center) within a specified dead-band (DB) limit. In this section, first order sensitivity of the regulating point voltage, $V_{\mathrm{LC}}$ estimated by LDC scheme to voltage support DG response is derived. DG VAr limits can be controlled for ensuring voltage support in 
distribution systems. Therefore, the sensitivity associated with the change in voltage with respect to the change in reactive power injection, $\Delta V_{\mathrm{LC}} / \Delta Q_{\mathrm{DG}}$ is considered.

\section{A. Proposed Voltage Sensitivity}

From the variables of LDC, (1) is derived. The magnitude and phasor angle of transformer secondary bus voltage are $V_{\text {OLTC }}$ and $\alpha_{\text {OLTC }}$, magnitude and phasor angle of the branch current seen at substation transformer secondary are respectively $I$ and $\theta$, while the LDC settings are $R_{\mathrm{L}}$ and $X_{\mathrm{L}}$. The topology of a sample distribution system with substation OLTC is shown in Fig. 1, where the respective phasor diagram for $\theta$-lagging power factor case is shown in Fig. 2. In conventional notation, the sending end voltage magnitude, $E$ (i.e. $E=V_{\text {OLTC }}$ ), receiving end voltage magnitude, $V$ (i.e. $V=V_{\text {OLTC }+1}$ ), branch current magnitude, $I$ and branch impedance, $Z$ are used for analysis in the branch defined by substation secondary bus and the bus next to the substation secondary.

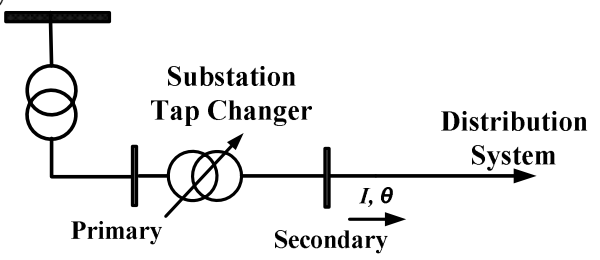

Fig. 1. Topology of distribution system with substation OLTC.

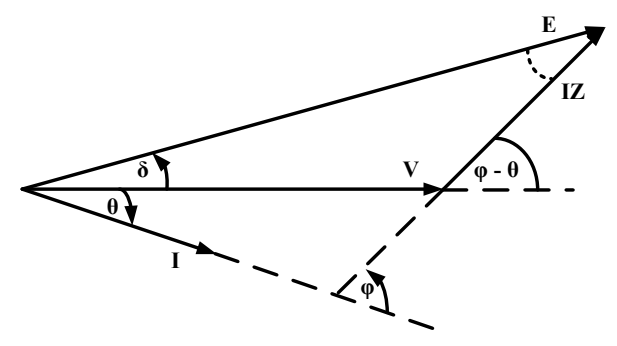

Fig. 2. Phasor diagram for $\theta$-lagging power factor case.

In this paper, the equations are presented only for $\theta$-lagging power factor case. For $\theta$-unity and leading power factor cases, similar analysis can be done.

$$
\begin{aligned}
& V_{L C}=\sqrt{\left(V_{\text {OLTC }}^{2}+I^{2} Z_{L}^{2}-2 V_{\text {OLTC }} I Z_{L} \cos \times\left(\theta-\varphi_{L}+\alpha_{\text {OLTC }}\right)\right)} \\
& \varphi_{L}=\tan ^{-1}\left(\frac{X_{L}}{R_{L}}\right), \quad Z_{L}=\sqrt{\left(R_{L}^{2}+X_{L}^{2}\right)}
\end{aligned}
$$

From the equation for $V_{\mathrm{LC}}$, it can be seen that the $V_{\mathrm{LC}}$ is a function of $V_{\text {OLTC }}, \alpha_{\text {OLTC }}, I, \theta, R_{\mathrm{L}}$ and $X_{\mathrm{L}}$ where $R_{\mathrm{L}}$ and $X_{\mathrm{L}}$ are constants.

$$
\begin{aligned}
& V_{L C}=f\left(V_{\text {OLTC }}, \alpha_{\text {OLTC }}, I, \theta, R_{L}, X_{L}\right) \\
& \Delta V_{L C}=\left(\frac{\partial V_{L C}}{\partial V_{\text {OLTC }}}\right) \Delta V_{\text {OLTC }}+\left(\frac{\partial V_{L C}}{\partial \alpha_{\text {OLTC }}}\right) \Delta \alpha_{\text {OLTC }}+\left(\frac{\partial V_{L C}}{\partial I}\right) \Delta I+ \\
& +\left(\frac{\partial V_{L C}}{\partial \theta}\right) \Delta \theta \\
& \Delta V_{L C}=(L C 1) \Delta V_{\text {OLTC }}+(L C 2) \Delta \alpha_{\text {OLTC }}+(L C 3) \Delta I+(L C 4) \Delta \theta
\end{aligned}
$$

The $L C 1$ to $L C 4$ terms in (1) can directly be obtained from the equation for $V_{\mathrm{LC}}$. In order to obtain the terms associated with $\Delta V_{\text {OLTC }}, \Delta \alpha_{\text {OLTC }}, \Delta I$, and $\Delta \theta$ in terms of small change in DG reactive power injection $\Delta Q_{\mathrm{DG}}$, firstly the expression given by (2) is derived using the phasor diagram shown in Fig. 2 and the cosine rule. The phasor angle of sending end voltage is $\alpha_{\mathrm{E}}$ (i.e. $\alpha_{\mathrm{E}}=\alpha_{\mathrm{OLTC}}$ ) and phasor angle of the receiving end voltage is $\alpha_{\mathrm{V}}$ (i.e. $\alpha_{E}=\alpha_{\mathrm{OLTC}+1}$ ).

$I=\frac{1}{Z} \sqrt{\left(E^{2}+V^{2}-2 E V \times \cos (\delta)\right)}$
$\left(\frac{\partial I}{\partial E}\right)=I 1, \quad\left(\frac{\partial I}{\partial V}\right)=I 2, \quad\left(\frac{\partial I}{\partial \delta}\right)=I 3$

$\Delta I=(I 1) \Delta E+(I 2) \Delta V+(I 3) \Delta \delta$

Secondly, from the same triangle which is used to derive (2) and applying the cosine rule, the equation (3) is derived.

$V=\sqrt{\left(I^{2} Z^{2}+E^{2}-2 I Z E \times \cos (\theta-\varphi+\delta)\right)}$

$\left(\frac{\partial V}{\partial I}\right)=\theta 1, \quad\left(\frac{\partial V}{\partial E}\right)=\theta 2, \quad\left(\frac{\partial V}{\partial \theta}\right)=\theta 3, \quad\left(\frac{\partial V}{\partial \delta}\right)=\theta 4$

$\Delta \theta=\left(\frac{1-\theta 1 . I 2}{\theta 3}\right) \Delta V-\left(\frac{\theta 2+\theta 1 . I 1}{\theta 3}\right) \Delta E-\left(\frac{\theta 4+\theta 1 . I 3}{\theta 3}\right) \Delta \delta$

Thirdly, the $\Delta V_{\mathrm{LC}} / \Delta Q_{\mathrm{DG}}$ sensitivity is derived using the change of voltage and current magnitudes and their phasor angles in the branch defined by substation secondary bus and the bus next to the substation secondary for small change in DG reactive power response, which can be obtained using the system Jacobian matrix as given below. They are voltage magnitude change for small change in DG reactive power response at the substation secondary bus, $\left(M_{V Q}\right)_{\text {oltc, } \mathrm{k}}$ and the bus next to the substation secondary, $\left(M_{V Q}\right)_{\text {oltc }+1, \mathrm{k}}$; and voltage phasor angle change for small change in DG reactive power response at the substation secondary bus, $\left(M_{\alpha Q}\right)_{\text {oltc, } \mathrm{k}}$ and the bus next to the substation secondary, $\left(M_{\alpha Q}\right)_{\text {oltc }+1, \mathrm{k}}$. It is assumed that the DG unit is connected to the $k^{\text {th }}$ bus of $n$ bus system.

$$
\begin{aligned}
& \frac{\Delta V_{\text {OLTC }}}{\Delta Q_{D G}}=\left(M_{V Q}\right)_{\text {oltc }, k}, \quad \frac{\Delta \alpha_{\text {OLTC }}}{\Delta Q_{D G}}=\left(M_{\alpha Q}\right)_{\text {oltc }, k} \\
& \frac{\Delta I}{\Delta Q_{D G}}=(I 1) \times\left(M_{V Q}\right)_{\text {oltc }, k}+(I 2) \times\left(M_{V Q}\right)_{(\text {oltc }+1), k}+(I 3) \times \\
& \times\left(\left(M_{\alpha Q}\right)_{\text {oltc }, k}-\left(M_{\alpha Q}\right)_{(\text {oltc }+1), k}\right) \\
& \frac{\Delta \theta}{\Delta Q_{D G}}=\left(\frac{1-\theta 1 . I 2}{\theta 3}\right) \times\left(M_{V Q}\right)_{(\text {oltc }+1), k}-\left(\frac{\theta 2+\theta 1 . I 1}{\theta 3}\right) \times \\
& \times\left(M_{V Q}\right)_{\text {oltc }, k}-\left(\frac{\theta 4+\theta 1.13}{\theta 3}\right) \times\left(\left(M_{\alpha Q}\right)_{\text {oltc }, k}-\left(M_{\alpha Q}\right)_{(o l t c+1), k}\right)
\end{aligned}
$$

Finally, the $\Delta V_{\mathrm{LC}} / \Delta Q_{\mathrm{DG}}$ sensitivity is derived by substituting $\Delta V_{\mathrm{OLTC}} / \Delta Q_{\mathrm{DG}}, \Delta \alpha_{\mathrm{OLTC}} / \Delta Q_{\mathrm{DG}}, \Delta I / \Delta Q_{\mathrm{DG}}$ and $\Delta \theta / \Delta Q_{\mathrm{DG}}$ terms to equation (1), and it is given by (4).

The detail derivations are shown in Appendix-I. 


$$
\begin{aligned}
& \frac{\Delta V_{L C}}{\Delta Q_{D G}}=(L C 1) \times\left(M_{V Q}\right)_{o l t c, k}+(L C 2) \times\left(M_{\alpha Q}\right)_{o l t c, k}+ \\
& +(L C 3) \times\left(\frac{\Delta I}{\Delta Q_{D G}}\right)+(L C 4) \times\left(\frac{\Delta \theta}{\Delta Q_{D G}}\right)
\end{aligned}
$$

For a case with multiple DG units distributed to a larger extent (i.e. $1, \ldots, g$ ), the superposition law can be applied to derive the respective sensitivities as given by (5).

$$
\begin{aligned}
& \frac{\Delta V_{L C}}{\Delta Q_{D G}}=\frac{\Delta V_{L C}}{\Delta Q_{D G 1}}+\frac{\Delta V_{L C}}{\Delta Q_{D G 2}}+\frac{\Delta V_{L C}}{\Delta Q_{D G 3}}+\frac{\Delta V_{L C}}{\Delta Q_{D G}}+\ldots \ldots \ldots . \\
& +\ldots \ldots \ldots .+\frac{\Delta V_{L C}}{\Delta Q_{D G}(g-1)}+\frac{\Delta V_{L C}}{\Delta Q_{D G g}}
\end{aligned}
$$

\section{B. Application Example for Testing the Proposed Method of Determining Voltage Sensitivity}

The proposed methodology is tested on a five-bus test system given in [9]. The sensitivity values are numerically obtained for different cases through simulations.

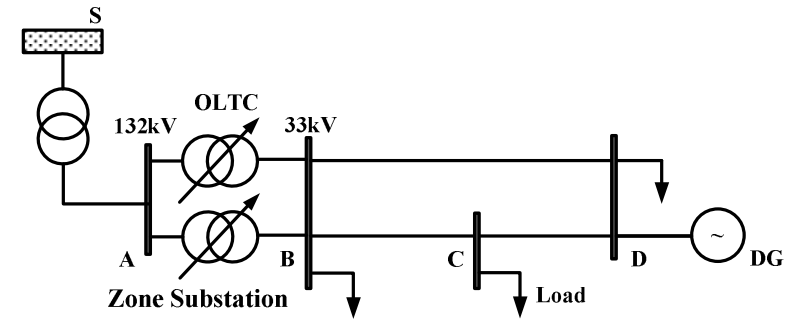

Fig. 3. Five bus test system.

The test cases include (a) testing proposed sensitivity assuming that the load centre is exactly at bus-C where LDC settings $\left(R_{\mathrm{L}}, X_{\mathrm{L}}\right)$ equal to resistance $(R)$ and reactance $(X)$ values of the branch between ' $\mathrm{B}$ ' and ' $\mathrm{C}$ ' and (b) testing proposed sensitivity assuming that the load centre is at bus-B where $R_{\mathrm{L}}=0$ and $X_{\mathrm{L}}=0$. For all simulation case studies, the substation secondary voltage is kept equal to $1.0 \mathrm{pu}$.

The results for case (a) are given in Table I and Table II. It can be seen that the maximum value of magnitude of mismatch between the sensitivity values derived using proposed method and direct method is $2.9 \%$ for simulated different system operations. The variation of sensitivity values derived using proposed and direct methods are shown in Fig. 4 and Fig. 5.

TABLE I

\begin{tabular}{c|c|c|c}
\multicolumn{2}{c}{ RESULTS FOR DGP $=25$ MW AND DGQ $=(-) 6.6$ MVAR - ABSORBING VAR } \\
\hline Load Factor & $\begin{array}{c}\text { Proposed } \\
\text { method }\end{array}$ & $\begin{array}{c}\text { Direct } \\
\text { method }\end{array}$ & $\begin{array}{c}\mid \text { Mismatch } \\
/(\%)\end{array}$ \\
\hline 1.000 & 0.2775 & 0.2837 & 2.2 \\
\hline 0.900 & 0.2735 & 0.2798 & 2.3 \\
\hline 0.800 & 0.2699 & 0.2763 & 2.3 \\
\hline 0.700 & 0.2667 & 0.2721 & 2.0 \\
\hline 0.600 & 0.2644 & 0.2702 & 2.1 \\
\hline 0.500 & 0.2625 & 0.2675 & 1.9 \\
\hline 0.400 & 0.2612 & 0.2653 & 1.5 \\
\hline 0.300 & 0.2605 & 0.2632 & 1.0 \\
\hline
\end{tabular}

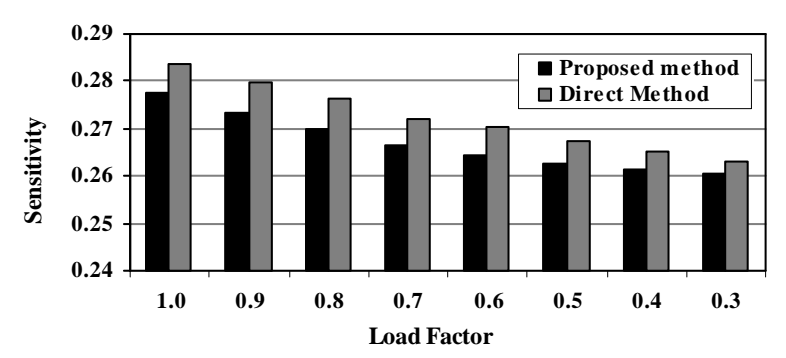

Fig. 4. Variation of sensitivity values derived using proposed and direct methods (Table I data).

TABLE II

RESULTS FOR DGP $=25 \mathrm{MW}$ AND DGQ $=(+) 6.6 \mathrm{MVAR}-$ EXPORTING VAR

\begin{tabular}{c|c|c|c}
\hline Load Factor & $\begin{array}{c}\text { Proposed } \\
\text { method }\end{array}$ & $\begin{array}{c}\text { Direct } \\
\text { method }\end{array}$ & $\begin{array}{c}\mid \text { Mismatch } \\
/(\%)\end{array}$ \\
\hline 1.000 & 0.2619 & 0.2544 & 2.9 \\
\hline 0.900 & 0.2588 & 0.2514 & 2.9 \\
\hline 0.800 & 0.2559 & 0.2487 & 2.9 \\
\hline 0.700 & 0.2551 & 0.2482 & 2.8 \\
\hline 0.600 & 0.2460 & 0.2440 & 0.8 \\
\hline 0.500 & 0.2438 & 0.2420 & 0.7 \\
\hline 0.400 & 0.2410 & 0.2403 & 0.3 \\
\hline 0.300 & 0.2380 & 0.2386 & 0.3 \\
\hline
\end{tabular}

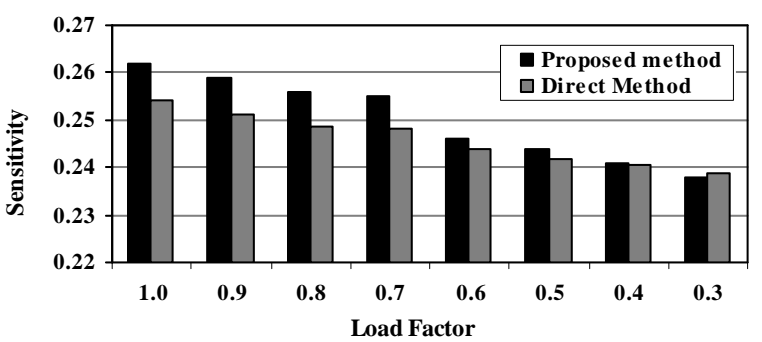

Fig. 5. Variation of sensitivity values derived using proposed and direct methods (Table II data).

The results for case (b) are given in Table III and Table IV for the same simulated system operations as in case (a). In this case study, it can be seen that the magnitude of mismatch between the sensitivity values derived using proposed method and direct method is zero for all simulated system operations.

TABLE III

RESULTS FOR DGP $=25$ MW AND DGQ = (-) 6.6 MVAR (ABSORBING VAR)

\begin{tabular}{c|c|c|c}
\hline Load Factor & $\begin{array}{c}\text { Proposed } \\
\text { method }\end{array}$ & $\begin{array}{c}\text { Direct } \\
\text { method }\end{array}$ & $\begin{array}{c}\mid \text { Mismatch } \mid \\
/(\%)\end{array}$ \\
\hline 1.000 & 0.2048 & 0.2048 & 0 \\
\hline 0.900 & 0.2016 & 0.2016 & 0 \\
\hline 0.800 & 0.1987 & 0.1987 & 0 \\
\hline 0.700 & 0.1961 & 0.1961 & 0 \\
\hline 0.600 & 0.1938 & 0.1938 & 0 \\
\hline 0.500 & 0.1918 & 0.1918 & 0 \\
\hline 0.400 & 0.1899 & 0.1899 & 0 \\
\hline 0.300 & 0.1883 & 0.1883 & 0 \\
\hline
\end{tabular}

In summary, test results have revealed the accuracy of the mathematical model used for deriving the proposed sensitivity. Hence, the $\Delta V_{\mathrm{LC}} / \Delta Q_{\mathrm{DG}}$ sensitivity can be used to accurately estimate the voltage change by DG voltage support operation at the fictitious load centre defined for LDC. Accuracy of such 
estimation is also tested for different system operations and the results are shown in Table $\mathrm{V}$ and Table VI.

TABLE IV

RESULTS FOR DGP $=25 \mathrm{MW}$ AND DGQ $=(+) 6.6 \mathrm{MVAR}($ EXPORTING VAR)

\begin{tabular}{c|c|c|c}
\hline Load Factor & $\begin{array}{c}\text { Proposed } \\
\text { method }\end{array}$ & $\begin{array}{c}\text { Direct } \\
\text { method }\end{array}$ & $\begin{array}{c}\mid \text { Mismatch } \\
/(\%)\end{array}$ \\
\hline 1.000 & 0.1828 & 0.1828 & 0 \\
\hline 0.900 & 0.1804 & 0.1804 & 0 \\
\hline 0.800 & 0.1782 & 0.1782 & 0 \\
\hline 0.700 & 0.1762 & 0.1762 & 0 \\
\hline 0.600 & 0.1744 & 0.1744 & 0 \\
\hline 0.500 & 0.1729 & 0.1729 & 0 \\
\hline 0.400 & 0.1715 & 0.1715 & 0 \\
\hline 0.300 & 0.1703 & 0.1703 & 0 \\
\hline
\end{tabular}

TABLE V

RESULTS FOR DGP $=25 \mathrm{MW}, \mathrm{DGQ}=(-) 6.6 \mathrm{MVAR}$ AND LOAD CENTER IS AT BUS-C (ABSORBING VAR)

\begin{tabular}{c|c|c|c}
\hline Load Factor & $\begin{array}{c}\text { Proposed } \\
\text { method/(pu) }\end{array}$ & $\begin{array}{c}\text { Multiple Power } \\
\text { Flow/(pu) }\end{array}$ & $\begin{array}{c}\mid \text { Mismatch } \\
/(\mathrm{pu})\end{array}$ \\
\hline 1.000 & 0.0183 & 0.0182 & 0.0001 \\
\hline 0.900 & 0.0181 & 0.0179 & 0.0002 \\
\hline 0.800 & 0.0178 & 0.0177 & 0.0001 \\
\hline 0.700 & 0.0176 & 0.0174 & 0.0002 \\
\hline 0.600 & 0.0175 & 0.0173 & 0.0002 \\
\hline 0.500 & 0.0173 & 0.0171 & 0.0002 \\
\hline 0.400 & 0.0172 & 0.0170 & 0.0002 \\
\hline 0.300 & 0.0172 & 0.0169 & 0.0003 \\
\hline
\end{tabular}

TABLE VI

RESULTS FOR DGP $=25 \mathrm{MW}, \mathrm{DGQ}=(+) 6.6 \mathrm{MVAR}$ AND LOAD CENTER IS AT BUS-C (EXPORTING VAR)

\begin{tabular}{c|c|c|c}
\hline Load Factor & $\begin{array}{c}\text { Proposed } \\
\text { method/(pu) }\end{array}$ & $\begin{array}{c}\text { Multiple Power } \\
\text { Flow/(pu) }\end{array}$ & $\begin{array}{c}\mid \text { Mismatch } \\
/(\mathrm{pu})\end{array}$ \\
\hline 1.000 & 0.0173 & 0.0172 & 0.0001 \\
\hline 0.900 & 0.0171 & 0.0171 & 0.0000 \\
\hline 0.800 & 0.0169 & 0.0169 & 0.0000 \\
\hline 0.700 & 0.0168 & 0.0171 & 0.0003 \\
\hline 0.600 & 0.0162 & 0.0165 & 0.0003 \\
\hline 0.500 & 0.0161 & 0.0164 & 0.0003 \\
\hline 0.400 & 0.0159 & 0.0163 & 0.0004 \\
\hline 0.300 & 0.0157 & 0.0161 & 0.0004 \\
\hline
\end{tabular}

According to the results shown in Tables V and VI, it can be seen that the estimated value of voltage change by DG at the load centre, which is derived using proposed sensitivity can be used to assess the impact of voltage support DG on operation of LDC.

\section{DyNAMIC ADJUSTMENT OF OLTC PARAMETERS}

The severity of DG impact on operation of LDC is based on how much the voltage change by DG at load centre contributes to violate the DB limit or supports to minimize the voltage error for OLTC control. On that basis, the proposed strategy for dynamically updating the LDC settings in presence of voltage support DG is developed. It is proposed to practically implement this strategy using a substation centered DMS for on-line voltage control [7]. Additional software and hardware required for enacting the proposed strategy for dynamically updating LDC settings is embedded in the DMS. The diagrammatic representation of proposed system design is shown in Fig. 6.

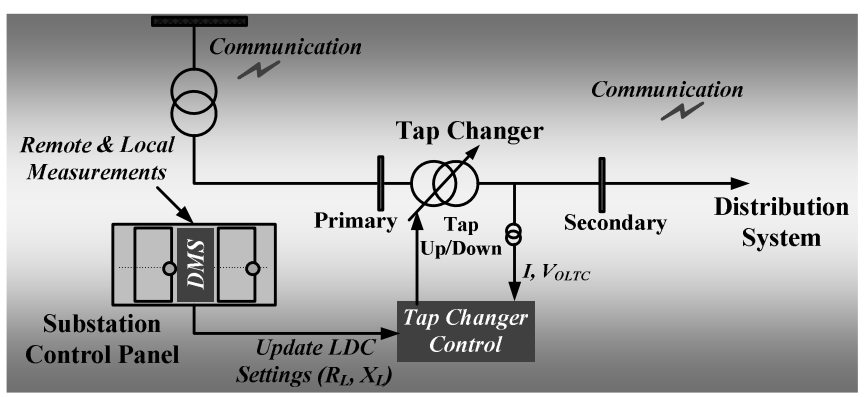

Fig. 6. Topology of system design for proposed dynamic operation of OLTC.

\section{A. Algorithm}

The algorithm of proposed strategy is outlined below. The objective of updating LDC settings is based on minimizing the number of OLTC tap operations and thereby minimizing the system voltage variations attributed to tap operations.

Step - 1: Estate estimation and power flow information from DMS are executed.

Step-2: Requirement of updating LDC settings is determined as given below. The set voltage for OLTC control is $V_{\text {set. }}$ The magnitude of difference between $V_{\text {set }}$ and $V_{\mathrm{LC}}$ is $V_{\text {err }}$.

$V_{\text {err }}=\left|V_{\text {set }}-V_{L C}\right|$

UpdateLDCsettings $\succ\left\{\begin{array}{cc}\text { Go toStep- } 3 & \text { if } V_{\text {err }}>D B / 2 \\ \text { Go toStep- } 1 & \text { Otherwise }\end{array}\right.$

Step - 3: Proposed $\Delta V_{\mathrm{LC}} / \Delta Q_{\mathrm{DG}}$ sensitivity value is derived using initial $R_{\mathrm{L}}$ and $X_{\mathrm{L}}$ settings.

Step - 4: New value for $\Delta V_{\mathrm{LC}} / \Delta Q_{\mathrm{DG}}$ sensitivity is defined using parameter $K_{\text {update, }}$ which minimizes the $V_{\text {err }}$ where system voltage is controlled within stipulated limits. A hysteresis tolerance value, $\varepsilon(+/-)$ is introduced to compensate the error in estimating the voltage change by $\mathrm{DG}$ using the $\Delta V_{\mathrm{LC}} / \Delta Q_{\mathrm{DG}}$ sensitivity. Next, the new $R_{\mathrm{L}}$ and $X_{\mathrm{L}}$ settings for LDC are derived and assigned.

$$
\left(\frac{\Delta V_{L C}}{\Delta Q_{D G}}\right)_{\text {new }}=\left\{K_{\text {update }} \times\left(\frac{\Delta V_{L C}}{\Delta Q_{D G}}\right)\right\}+\in
$$

Step - 5: If there is no feasible solution in Step-4 for new $R_{\mathrm{L}}$ and $X_{\mathrm{L}}$ settings, their initial values are assigned.

Step - 6: For the subsequent state (i.e. $t=t+1$ ), repeat the procedure from Step -1 .

\section{B. Time Domain Simulation}

Simulations are used for testing the proposed strategy. It is assumed that the LDC settings are conventionally derived using the method given in [11]. The initial time delay, $T_{\mathrm{d}}$, mechanical time delay, $T_{\mathrm{m}}$ and DB of the tap changer are $30 \mathrm{~s}$, $3 \mathrm{~s}$ and $1.0 \%$, respectively. It is assumed that the OLTC taps are incorporated in primary winding of the substation transformer. Assumed system voltage bandwidth is $10 \% \mathrm{pu}$. 
The following power flow simulation elaborates the proposed algorithm and its mechanism for dynamically updating the LDC settings in presence of synchronous machine based voltage support DG. A representative state of 96.936 MVA (peak) step load is considered assuming that the initial OLTC tap is at its nominal position. The synchronous machine based DG unit supplies $25 \mathrm{MW}$ power to the system where reactive power support provided by DG for voltage support is 6.6 MVAr. The conventionally derived LDC settings are $R_{\mathrm{L}}=$ $5.003 \Omega(\sim 5.0 \Omega)$ and $X_{\mathrm{L}}=2.121 \Omega(\sim 2.1 \Omega)$. The values of $V_{\text {set }}, V_{\text {err }}$ and $\Delta V_{\mathrm{LC}} / \Delta Q_{\mathrm{DG}}$ sensitivity are $1.0000 \mathrm{pu}, 0.0561 \mathrm{pu}$ $\left(V_{\text {err }}>\mathrm{DB} / 2=0.0050 \mathrm{pu}\right)$ and 3.1345 , respectively. The value assigned for $\varepsilon$ is zero. Fig. 6 shows the number of tap operations and resultant voltage variations at substation secondary bus and bus-C.

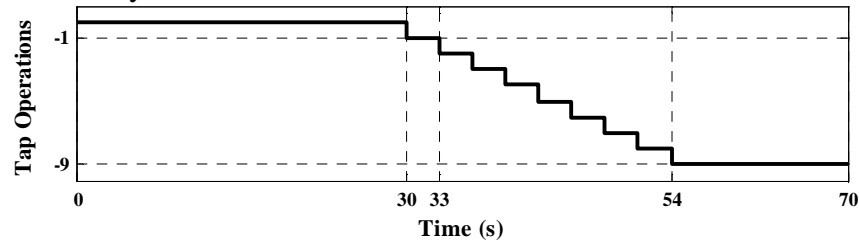

(a)

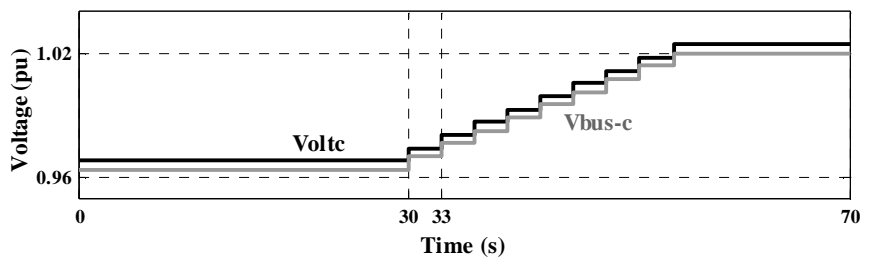

(b)

Fig. 6. (a) Tap operations of OLTC and (b) voltage at substation transformer secondary bus and bus-C, under conventionally derived LDC settings.

In this case, the estimated value of voltage change at load centre by voltage support DG is 0.2069 pu. However, this voltage change by DG does not lead to violate the DB limit, because the $V_{\text {err }}$ without DG reactive power support is 0.0728 pu. Therefore, first a new set of LDC settings is searched using $K_{\text {update }}>1$. If there is not a feasible solution for $K_{\text {update }}>1$, new LDC settings are searched using $K_{\text {update }}<1$. In this case, the new $R_{\mathrm{L}}$ and $X_{\mathrm{L}}$ settings are derived using $K_{\text {update }}$ value of $0.4400(<1.0000)$ assuming that the initial settings are equal to conventional settings, and they are $R_{\mathrm{L}}=1.15 \Omega(\sim 1.2 \Omega)$ and $X_{\mathrm{L}}=2.31 \Omega(\sim 2.3 \Omega)$. For finding a value for $K_{\text {update, }}$ a quartic equation is solved for $Z_{\mathrm{L}}$ with assumed $\left(X_{\mathrm{L}} / R_{\mathrm{L}}\right)$ ratio. With the updated LDC settings, the $V_{\text {err }}$ is 0.0394 pu. Fig. 7 (a) shows the number of tap operations with new LDC settings which is less than that with conventionally tuned settings, while Fig. 7 (b) shows the variation in voltages. It is noted that in case where the voltage change by DG does lead to violate the DB limit, first a new set of LDC settings is searched using $K_{\text {update }}<1$. If there is not a feasible solution for $K_{\text {update }}<1$, new LDC settings are searched using $K_{\text {update }}>1$.

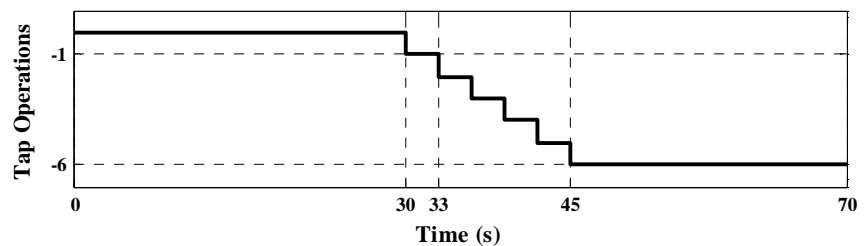

(a)

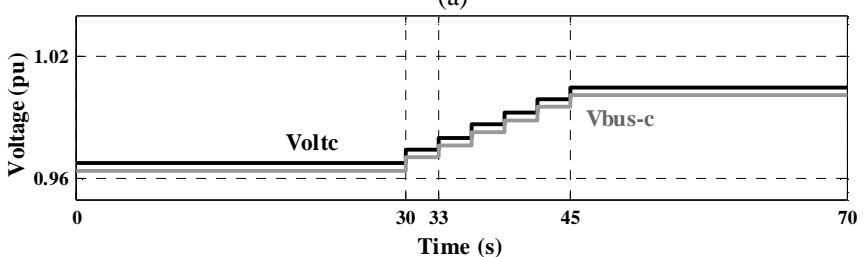

(b)

Fig. 7. (a) Tap operations of OLTC and (b) voltage at substation transformer secondary bus and bus-C, under proposed LDC settings.

\section{CONCLUSION}

A novel methodology is proposed in this paper to assess the impact of voltage support DG on voltage correction and update the OLTC parameters dynamically. As an assessment tool, first order sensitivity of the regulating point voltage to the change in reactive power support provided by voltage support DG is derived. The estimated value of voltage change by means of VAr support by DG at the load centre, obtained using proposed sensitivity analysis, is used to assess the impact of voltage support DG on the operation of LDC. Based on such information, a strategy is proposed to dynamically update the OLTC parameters for effective voltage control in the presence of voltage support DG. The simulation results show the accuracy of the mathematical model used for analysis and its successful implementation.

\section{REFERENCES}

[1] P. N. Vovos, A. E. Kiprakis, A. R. Wallace, and G. P. Harrision, "Centralized and Distributed Voltage Control: Impact on Distributed Generation Penetration," IEEE Trans. Power Systems, vol. 22, no. 1, pp. 476-483, Feb. 2007.

[2] H. S. Ko, G. G. Yoon, and W. P. Hong, "Active Use of DFIG-Based Variable-Speed Wind-Turbine for Voltage Regulation at a Remote Location," IEEE Trans. Power Systems, vol. 22, no. 4, pp. 476-483, Feb. 2007.

[3] J. Hu, H. Nian, B. Hu, Y. He, and Z. Q. Zhu, "Direct Active and Reactive Power Regulation of DFIG Using Sliding-Mode Control Approach," IEEE Trans. Energy Conversion, vol. 25, no.4, pp. 10281039, Dec. 2010.

[4] R. Aghatehrani, and R. Kavasseri, "Reactive Power Management of a DFIG Wind System in Micro-girds based on Voltage Sensitivity Analysis," IEEE Trans. Sustainable Energy, vol. 2, no. 4, pp. 451-458, Oct. 2011.

[5] K. Turitsyn, P. Sulc, S. Backhaus, and M. Chertkov, "Options for Control of Reactive Power by Distributed Photovoltaic Generators," Proceedings of the IEEE, vol. 99, pp. 1063-1073, June 2011.

[6] R. K. Varma, V. Khadkikar, and R. Seethapathy, "Night-time Application of PV Solar Farm as STATCOM to Regulate Grid Voltage," IEEE Trans. Energy Conversion, vol. 24, no. 4 pp. 983-985, Dec. 2009.

[7] D. Ranamuka, A. P. Agalgaonkar, and K. M. Muttaqi, "On-line Voltage Control in Distribution Systems with Multiple Voltage Regulating Devices," accepted for publication in IEEE Trans. Sustainable Energy.

[8] K. Zou, A. P. Agalgaonkar, K. M. Muttaqi, and S. Perera, "Distribution System Planning With Incorporating DG Reactive Capability and System Uncertainties," IEEE Trans. Sustainable Energy, vol. 3, no. 1, pp. 112-123, Jan. 2012. 
[9] N. Jenkins, R. Allan, P. Crossley, D. Kirschen and G. Strbac, Embedded Generation, vol. 1. London: Cambridge University Press, 2000, pp. 4963.

\section{APPENDIX-I}

$$
\begin{aligned}
& L C 1=\frac{\partial V_{L C}}{\partial V_{\text {OLTC }}}=\frac{\left(V_{\text {OLTC }}-I \cdot Z_{L} \cdot \cos \left(\theta-\phi_{L}+\alpha_{O L T C}\right)\right)}{\sqrt{\left(V_{\text {OLTC }}^{2}+I^{2} \cdot Z_{L}^{2}-2 \cdot V_{O L T C} \cdot I \cdot Z_{L} \cdot \cos \left(\theta-\phi_{L}+\alpha_{O L T C}\right)\right.}}, \quad L C 2=\frac{\partial V_{L C}}{\partial \alpha_{O L T C}}=\frac{V_{O L T C} \cdot I \cdot Z_{L} \cdot \sin \left(\theta-\phi_{L}+\alpha_{O L T C}\right)}{\sqrt{\left(V_{O L T C}{ }^{2}+I^{2} \cdot Z_{L}^{2}-2 \cdot V_{O L T C} \cdot I \cdot Z_{L} \cdot \cos \left(\theta-\phi_{L}+\alpha_{O L T C}\right)\right.}} \\
& L C 3=\frac{\partial V_{L C}}{\partial I}=\frac{\left(I \cdot Z_{L}{ }^{2}-V_{O L T C} \cdot Z_{L} \cdot \cos \left(\theta-\phi_{L}+\alpha_{O L T C}\right)\right)}{\sqrt{\left(V_{O L T C}{ }^{2}+I^{2} \cdot Z_{L}^{2}-2 \cdot V_{O L T C} \cdot I \cdot Z_{L} \cdot \cos \left(\theta-\phi_{L}+\alpha_{O L T C}\right)\right)}}, \quad L C 4=\frac{\partial V_{L C}}{\partial \theta}=\frac{V_{O L T C} \cdot I \cdot Z_{L} \cdot \sin \left(\theta-\phi_{L}+\alpha_{O L T C}\right)}{\sqrt{\left(V_{O L T C}{ }^{2}+I^{2} \cdot Z_{L}{ }^{2}-2 \cdot V_{O L T C} \cdot I \cdot Z_{L} \cdot \cos \left(\theta-\phi_{L}+\alpha_{O L T C}\right)\right)}} \\
& I 1=\left(\frac{\partial I}{\partial E}\right)=\frac{E-V \cdot \cos (\delta)}{Z \times \sqrt{\left(E^{2}+V^{2}-2 \times E \times V \times \cos (\delta)\right)}}, \quad I 2=\left(\frac{\partial I}{\partial V}\right)=\frac{V-E \cdot \cos (\delta)}{Z \times \sqrt{\left(E^{2}+V^{2}-2 \times E \times V \times \cos (\delta)\right)}}, \quad I 3=\left(\frac{\partial I}{\partial \delta}\right)=\frac{E V \cdot \sin (\delta)}{Z \times \sqrt{\left(E^{2}+V^{2}-2 \times E \times V \times \cos (\delta)\right)}} \\
& \theta 1=\left(\frac{\partial V}{\partial I}\right)=\frac{Z(I Z-E \cdot \cos (\delta))}{\sqrt{\left(I^{2} Z^{2}+E^{2}-2 \times I \times Z \times E \times \cos (\theta-\phi+\delta)\right)}}, \quad \theta 2=\left(\frac{\partial V}{\partial E}\right)=\frac{E-I Z \cdot \cos (\delta)}{\sqrt{\left(I^{2} Z^{2}+E^{2}-2 \times I \times Z \times E \times \cos (\theta-\phi+\delta)\right.}} \\
& \theta 3=\left(\frac{\partial V}{\partial \theta}\right)=\frac{I Z E \cdot \sin (\theta-\phi+\delta)}{\sqrt{\left(I^{2} Z^{2}+E^{2}-2 \times I \times Z \times E \times \cos (\theta-\phi+\delta)\right)}}, \quad \theta 4=\left(\frac{\partial V}{\partial \delta}\right)=\frac{I Z E \cdot \sin (\theta-\phi+\delta)}{\sqrt{\left(I^{2} Z^{2}+E^{2}-2 \times I \times Z \times E \times \cos (\theta-\phi+\delta)\right.}} \\
& \frac{\Delta V_{L C}}{\Delta Q_{D G}}=(L C 1) \times\left(M_{V Q}\right)_{\text {oltc }, k}+(L C 2) \times\left(M_{\alpha Q}\right)_{\text {oltc }, k}+(L C 3) \times\left((I 1) \times\left(M_{V Q}\right)_{\text {oltc }, k}+(I 2) \times\left(M_{V Q}\right)_{\text {oltc }+1, k}+(I 3) \times\left(\left(M_{\alpha Q}\right)_{\text {oltc }, k}-\left(M_{\alpha Q}\right)_{\text {oltc }+1, k}\right)\right)+ \\
& +(L C 4) \times\left(\left(\frac{1-\theta 1 . I 2}{\theta 3}\right) \times\left(M_{V Q}\right)_{\text {oltc }+1, k}-\left(\frac{\theta 2+\theta 1 . I 1}{\theta 3}\right) \times\left(M_{V Q}\right)_{\text {oltc }, k}-\left(\frac{\theta 4+\theta 1 . I 3}{\theta 3}\right) \times\left(\left(M_{\alpha Q}\right)_{\text {oltc }, k}-\left(M_{\alpha Q}\right)_{\text {oltc }+1, k}\right)\right)
\end{aligned}
$$

